- Original Article

\title{
Concurrence of Helicobacter pylori Infection and Its Associated Factors in Korean Couples
}

\author{
Jiwoong Choi, Jihun Kang* \\ Department of Family Medicine, Kosin University Gospel Hospital, Busan, Korea
}

Background: This study evaluated the prevalence of Helicobacter pylori infection and the risk factors associated with $H$. pylori transmission among spouses.

Methods: We assessed the spousal concurrence of $H$. pylori infection using the Campylobacter-like organism (CLO) test under gastro-endoscopy in 132 couples. Based on the CLO test results, participants were categorized into $H$. pylori concurrent and independent groups. The chi-square test and Student t-test were performed for demographic comparisons between the concurrent and independent $H$. pylori groups. In addition, multivariate logistic regression analysis was performed to identify factors associated with concurrent $H$. pylori infection.

Results: The study revealed that the concurrence rate of $H$. pylori infection was $42.4 \%$ in married Korean couples. The odds ratio (OR) derived from the concurrence of $H$. pylori infection tended to decrease in older couples (OR, 0.975; 95\% confidence interval [CI], 0.949-1.002; $\mathrm{P}=0.072$ ). Gastric erosion was also associated with a decreased OR for concurrent infection (OR, 0.488; 95\% CI, 0.295-0.808; $\mathrm{P}=0.005)$. Conversely, active duodenal ulcers were associated with an increased OR for concurrent infections (OR, 6.501; 95\% CI, 1.267-33.346; $\mathrm{P}=0.025$ ). Duodenal ulcer scars tended to increase the $\mathrm{OR}$ of concurrent infections ( $\mathrm{OR}, 1.392 ; 95 \% \mathrm{CI}, 0.815-2.380 ; \mathrm{P}=0.226$ ).

Conclusion: Spousal transmission and concurrence of $H$. pylori infection were negatively associated with gastric erosion; however, they were positively associated with active duodenal ulcers. Further studies are warranted to elucidate the mechanisms underlying these findings.

Keywords: Helicobacter pylori; Spouses; Infectious Disease Transmission; Duodenal Ulcer

Received: June 17, 2021, Revised: July 16, 2021, Accepted: July 29, 2021

*Corresponding Author: Jihun Kang https://orcid.org/0000-0002-2263-9054

Tel: +82-51-990-3089, Fax: +82-51-990-3005, E-mail: josua85@naver.com 


\section{INTRODUCTION}

Helicobacter pylori is a gram-negative bacillus that exists between the gastric mucous layer and the gastric epithelial cells. ${ }^{1)}$ This bacillus is considered a major cause of various gastrointestinal diseases such as gastritis, gastric and duodenal ulcers, mucosa-associated lymphoid tissue lymphoma, and gastric cancer. Globally, approximately 4.4 billion individuals developed $H$. pylori infection in 2015. ${ }^{2)}$ In Korea, the number of individuals with $H$. pylori infection was estimated to be $13,571,123$, with a prevalence rate of $54.0 \%$ in $2015^{2)}$

A myriad of factors, including low socioeconomic status and poor hygiene, are associated with $H$. pylori infection. ${ }^{3)}$ However, the modes of transmission of $H$. pylori are still poorly understood. Person-to-person contact (fecal-oral and oral-oral) is considered the most common route of infection; ${ }^{4,5)}$ however, the disease may also be transmitted through contaminated water, ${ }^{4,5)}$ animal reservoirs, ${ }^{4,5)}$ and other environmental factors. ${ }^{4,5)}$ In addition, differences in patterns of household transmission and infection rates of $H$. pylori among geographic regions make it difficult to clearly determine the exact pathways of $H$. pylori infection.

Although parent-to-child transmission is the most common occurrence, conjugal transmission also plays a significant role in intra-familial transmission. An accumulating body of evidence indicates that $H$. pylori infection in one spouse is a major source of spread to other household members. ${ }^{6)}$ Singh et al. ${ }^{6}$ showed that the infection rate in spouses of $H$. pylori-positive individuals was significantly higher than that in $H$. pylori-negative spouses. Similarly, previous studies also support that the presence of an $H$. pylori-positive spouse is an independent risk factor for $H$. pylori infection in the other partner. ${ }^{7,8)}$

Despite the existing pool of evidence, it remains uncertain which specific factors are associated with the conjugal transmission of $H$. pylori, especially in Korean adults. Therefore, the present study evaluated the prevalence of $H$. pylori infection and the risk factors associated with concurrent $H$. pylori infection among spouses.

\section{METHODS}

\section{Study Population}

We screened 4,583 individuals who underwent the Campylobacterlike organism (CLO) test under gastroscopy at Kosin University Gospel Hospital from January 1, 2010, to December 31, 2019, to identify eligible participants. Among them, 4,319 individuals were excluded from the analysis: (1) individuals whose spouses did not have a record of undergoing a CLO test and those with a difference of 30 days or more in the dates that the person and their spouse performed their respective CLO tests (N=4,143); (2) individuals who had taken non-steroidal anti-inflammatory drugs, proton pump inhibitors, antibiotics, bismuth compounds, and mucosal protective drugs that may affect the CLO test results within the last three months of testing ( $\mathrm{N}=114)$; and (3) individuals who were $H$. pylori-negative in the CLO test for themselves and their spouses $(\mathrm{N}=62)$.

Following extensive screening, 264 participants (132 couples) were included in the final analysis.

The eligible participants were then classified into two groups: group A (the husband and wife were both $H$. pylori-positive [concurrence group]) and group B (only one spouse was $H$. pylori-positive [independence group]). The flow chart for the selection of the study participants is shown in Figure 1.

The requirement for informed consent from individual patients was waived because of the retrospective design of this study. All study protocols complied with the Declaration of Helsinki. This study was reviewed and approved by the Institutional Review Board of Kosin University Medical School (KUGH-2020-05-003).

\section{Helicobacter pylori Tests}

The participants underwent gastroscopy to confirm the presence of $H$. pylori-associated esophagogastroduodenal disease, and biopsies were obtained for the CLO test. After an overnight fast, the participants underwent gastroscopy with lidocaine administered as local anesthetic throughout the throat area. Some participants who needed conscious

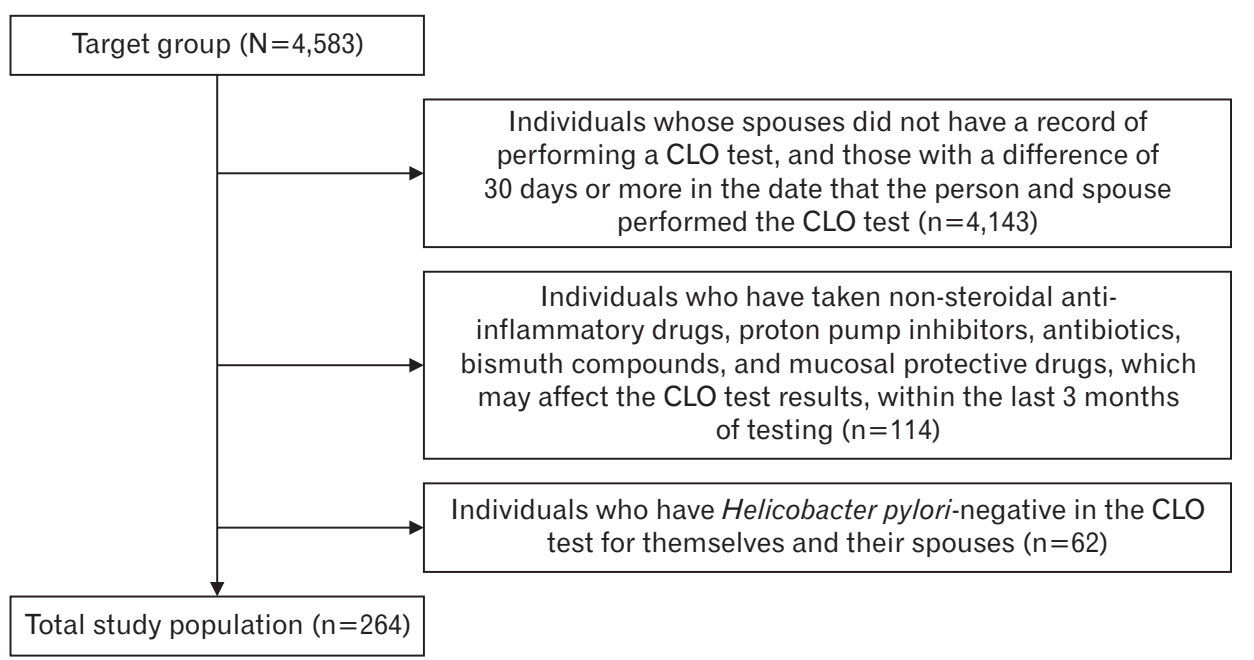

Figure 1. Flow diagram for study participants. CLO test, Campylobacter-like organism test. 
sedation were administered intravenous midazolam. Biopsy specimens were obtained from each participant's gross lesions in the stomach and duodenum using biopsy forceps. Tissues were cultured in a CLO test kit (Delta West, Bentley, Australia), and the results were read within 2 hours. If the color of the culture medium changed from yellow to red, it was considered $H$. pylori-positive; however, if it remained yellow, the finding was considered negative. In particular, whenever a negative result was acquired within the 2 hours, the final reading was performed again after 24 hours.

\section{Data Collection and Measurements}

Information on demographic variables (age), socioeconomic status (education and occupation), lifestyle habits (smoking status and alcohol consumption), and gastrointestinal symptoms were obtained using a self-administered questionnaire at the time of visit for their health check-up. Upon consideration of aspects such as educational qualification (high school and college or higher), occupation (manual workers: fishermen, armed forces personnel, technical workers, production workers, and construction workers; non-manual workers: housewives, managers, service and sales workers, religious workers, politicians, medical workers, transport workers, white-collar workers, education workers, and unemployed individuals), smoking status (non-smokers: never and ex-smokers; and smokers: current smokers), and alcohol consumption status, the participants were categorized

Table 1. General characteristics of participants

\begin{tabular}{|c|c|c|c|c|}
\hline Characteristic & $\begin{array}{l}\text { Total participants } \\
\quad(\mathrm{n}=264)\end{array}$ & $\begin{array}{l}\text { Independence H. pylori infection } \\
\qquad(\mathrm{n}=152)\end{array}$ & $\begin{array}{l}\text { Concurrence H. pylori infection } \\
\qquad(\mathrm{n}=112)\end{array}$ & P-value* \\
\hline Age (y) & $48.6 \pm 10.5$ & $49.7 \pm 10.6$ & $47.1 \pm 10.3$ & 0.069 \\
\hline Body mass index $\left(\mathrm{kg} / \mathrm{m}^{2}\right)$ & $23.8 \pm 3.4$ & $22.6 \pm 2.8$ & $25.0 \pm 3.6$ & 0.595 \\
\hline Education & & & & 0.599 \\
\hline High school & $22(8.3)$ & $11(7.2)$ & $11(9.8)$ & \\
\hline College or above & $242(91.7)$ & $141(92.8)$ & $101(90.2)$ & \\
\hline Occupation & & & & 0.268 \\
\hline Non-manual workers & $210(79.5)$ & $125(82.2)$ & 85 (75.9) & \\
\hline Manual workers & $54(20.5)$ & $27(17.8)$ & $27(24.1)$ & \\
\hline Alcohol consumption & & & & 1.000 \\
\hline No & $101(38.3)$ & 58 (38.2) & $43(38.4)$ & \\
\hline Yes & $163(61.7)$ & $94(61.8)$ & $69(61.6)$ & \\
\hline Smoking & & & & 0.927 \\
\hline Non-smokers & $168(63.6)$ & $98(64.5)$ & $70(62.5)$ & \\
\hline Smokers & $96(36.4)$ & $54(35.5)$ & $42(37.5)$ & \\
\hline \multicolumn{5}{|l|}{ Endoscopic findings } \\
\hline Gastric erosion & $155(58.7)$ & $101(66.5)$ & $54(48.2)$ & 0.004 \\
\hline Non-atropic gastritis & $103(39.0)$ & $60(39.5)$ & $43(38.4)$ & 0.960 \\
\hline Atropic gastritis & $142(53.8)$ & $76(50.0)$ & $66(58.9)$ & 0.189 \\
\hline Gastric polyp & $16(6.1)$ & $9(5.9)$ & $7(6.3)$ & 1.000 \\
\hline Reflux esophagitis & $75(28.4)$ & $48(31.6)$ & $27(24.1)$ & 0.233 \\
\hline Gastric benign tumor & $3(1.1)$ & $1(0.7)$ & $2(1.8)$ & 0.789 \\
\hline Gastric malignancy & $3(1.1)$ & $1(1.3)$ & $2(1.8)$ & 1.000 \\
\hline Gastric ulcer & $70(26.5)$ & $43(28.3)$ & $27(24.1)$ & 0.535 \\
\hline Active duodenal ulcer & $9(3.4)$ & $2(1.3)$ & $7(6.3)$ & 0.066 \\
\hline Duodenal ulcer scar & $164(62.1)$ & $91(59.9)$ & $73(65.2)$ & 0.453 \\
\hline \multicolumn{5}{|l|}{ Gastrointestinal symptoms } \\
\hline Nausea & $13(4.9)$ & $9(5.9)$ & $4(3.6)$ & 0.559 \\
\hline Epigastric soreness & $28(10.6)$ & $17(11.2)$ & $11(9.8)$ & 0.878 \\
\hline Indigestion & $44(16.7)$ & 25 (16.5) & $19(17.0)$ & 1.000 \\
\hline Weight loss & $5(1.9)$ & $3(2.0)$ & $2(1.8)$ & 1.000 \\
\hline \multicolumn{5}{|l|}{ Concomitant diseases } \\
\hline Hypertension & 64 (24.2) & 48 (31.6) & $16(14.3)$ & 0.263 \\
\hline Diabetes mellitus & $32(12.1)$ & 21 (13.8) & $11(9.8)$ & 0.743 \\
\hline \multicolumn{5}{|l|}{ Blood lab } \\
\hline Cholesterol (mg/dL) & $196.2 \pm 44.9$ & $193.7 \pm 41.7$ & $199.6 \pm 49.1$ & 0.295 \\
\hline Triglyceride (mg/dL) & $121.1 \pm 138.0$ & $110.7 \pm 65.9$ & $135.3 \pm 197.6$ & 0.209 \\
\hline $\mathrm{HDL}-\mathrm{C}(\mathrm{mg} / \mathrm{dL})$ & $54.7 \pm 14.5$ & $54.5 \pm 15.1$ & $55.0 \pm 13.7$ & 0.747 \\
\hline LDL-C (mg/dL) & $121.2 \pm 37.6$ & $121.0 \pm 39.2$ & $121.6 \pm 35.8$ & 0.904 \\
\hline
\end{tabular}

Values are presented as mean \pm standard deviation or number (\%).

H. pylori, Helicobacter pylori; HDL-C, high-density lipoprotein cholesterol; LDL-C, low-density lipoprotein cholesterol.

${ }^{*}$ Calculated using Student T-test for continuous variable and chi-square test for categorical variable. 
into two groups. Participants were then asked to report any gastrointestinal symptoms (including nausea, epigastric soreness, indigestion) they were experiencing at the time of the examination. Body weight and height were measured automatically, and the body mass index was calculated as body mass in kilograms divided by the height in meters squared.

Lipid profiles (total cholesterol, triglyceride, high-density lipoprotein cholesterol, and low-density lipoprotein cholesterol) were obtained using enzymatic methods after at least 8 hours of fasting. Data on hypertension and diabetes were obtained from the medical records. Finally, the endoscopic findings were determined by combining the gross findings of the operator and the histological results after specimen collection.

\section{Statistical Analysis}

The chi-square test and Student t-test were performed to determine the demographic comparison between the concurrent and independent $H$. pylori groups. Bivariate and multivariate logistic regression analyses were performed to identify numerous factors (occupation, age, body mass index, education level, physical labor, smoking history, drinking history, hypertension history, diabetes mellitus history, digestive symptoms, and endoscopy findings) associated with concurrent $H$. pylori infection. All statistical analyses were done using IBM SPSS Statistics software ver. 26.0 (IBM Corp., Armonk, NY, USA). Statistical significance was set at a two-tailed P-value of $<0.05$.

\section{RESULTS}

The study showed that the concurrence rate of $H$. pylori infection was $42.4 \%$ in married Korean couples. Table 1 presents a comparison of the general characteristics between the concurrent and independent groups. The mean ages of the independent and concurrent groups were $49.7 \pm 10.6$ years and $47.1 \pm 10.3$ years, respectively. Gastric erosion was also found to be higher in the independent group (66.5\%) than in the concurrent group (48.2\%). There were no significant differences in other characteristics, such as age, socioeconomic status, health behaviors, and biochemical variables between the two groups.

Table 2 shows the general characteristics associated with concurrent $H$. pylori infections. General characteristics were not significantly associated with the conjugal infection of $H$. pylori.

We used multivariate logistic regression analysis to test the gastrointestinal symptoms and endoscopic findings associated with concurrent $H$. pylori infections (Table 3). Gastric erosion was associated with a decreased odds ratio (OR) for concurrent infections (OR, 0.488; 95\% confidence interval [CI], 0.295-0.808; $\mathrm{P}=0.005)$. In contrast, active duodenal ulcers were associated with an increased OR for concurrent infections (OR, 6.501; 95\% CI, 1.267-33.346; $\mathrm{P}=0.025$ ). Although the presence of a duodenal ulcer scar tended to increase the OR of concurrent infections (OR, 1.392; 95\% CI, 0.815-2.380; $\mathrm{P}=0.226$ ).

\section{DISCUSSION}

This study showed that concurrent $H$. pylori infection was observed in

Table 2. General factors associated with concurrent Helicobacter pylori infection among spouses

\begin{tabular}{|c|c|c|c|c|}
\hline \multirow{2}{*}{ Variable } & \multicolumn{2}{|c|}{ Univariate analysis } & \multicolumn{2}{|c|}{ Multivariate analysis } \\
\hline & OR (95\% Cl) & P-value* & $\mathrm{OR}(95 \% \mathrm{Cl})$ & P-value* \\
\hline Age (y) & $0.978(0.956-1.002)$ & 0.070 & 0.975 (0.949-1.002) & 0.072 \\
\hline Body mass index & 1.019 (0.950-1.094) & 0.594 & $1.011(0.031-1.097)$ & 0.802 \\
\hline Education & & 0.454 & & 0.132 \\
\hline High school & Reference & & Reference & \\
\hline College or above & $1.396(0.583-3.345)$ & & $2.108(0.798-5.568)$ & \\
\hline Occupation & & 0.208 & & 0.283 \\
\hline Non-manual workers & Reference & & Reference & \\
\hline Manual workers & $0.680(0.373-1.240)$ & & $0.683(0.340-1.370)$ & \\
\hline Alcohol consumption & & 0.969 & & 0.477 \\
\hline No & Reference & & Reference & \\
\hline Yes & $1.010(0.611-1.669)$ & & $1.265(0.663-2.413)$ & \\
\hline Smoking & & 0.742 & & 1.028 \\
\hline Non-smoker & Reference & & Reference & \\
\hline Smokers & $1.089(0.656-1.807)$ & & $1.028(0.517-2.047)$ & \\
\hline Hypertension & & 0.202 & & 0.211 \\
\hline No & Reference & & Reference & \\
\hline Yes & $1.537(0.794-2.975)$ & & $1.625(0.760-3.475)$ & \\
\hline Diabetes mellitus & & 0.584 & & 0.616 \\
\hline No & Reference & & Reference & \\
\hline Yes & $0.787(0.334-1.855)$ & & 0.787 (0.309-2.004) & \\
\hline
\end{tabular}

$\mathrm{OR}$, odds ratio; $\mathrm{Cl}$, confidence interval.

${ }^{*}$ Calculated using single and multiple logistic regression. 
Table 3. Gastro-intestinal symptoms and endoscopic findings associated with concurrent Helicobacter pylori infection among spouses

\begin{tabular}{|c|c|c|}
\hline \multirow{2}{*}{ Variable } & \multicolumn{2}{|c|}{ Multivariate analysis } \\
\hline & Odds ratio (95\% Cl) & P-value* \\
\hline \multicolumn{3}{|c|}{ Gastrointestinal symptoms } \\
\hline Nausea & 0.589 (0.176-1.970) & 0.390 \\
\hline Epigastric soreness & $0.893(0.399-1.999)$ & 0.784 \\
\hline Indigestion & $0.914(0.466-1.793)$ & 0.795 \\
\hline Weight loss & $0.897(0.146-5.508)$ & 0.907 \\
\hline \multicolumn{3}{|l|}{ Endoscopic findings } \\
\hline Gastric erosion & $0.488(0.295-0.808)$ & 0.005 \\
\hline Non-atropic gastritis & $1.208(0.691-2.112)$ & 0.508 \\
\hline Atropic gastritis & $1.245(0.731-2.123)$ & 0.420 \\
\hline Gastric polyp & $1.175(0.418-3.306)$ & 0.759 \\
\hline Reflux esophagitis & $0.692(0.397-1.205)$ & 0.193 \\
\hline Gastric benign tumor & $3.695(0.320-42.657)$ & 0.295 \\
\hline Gastric malignancy & $1.588(0.217-11.612)$ & 0.649 \\
\hline Gastric ulcer & $0.895(0.504-1.589)$ & 0.704 \\
\hline Active duodenal ulcer & $6.501(1.267-33.346)$ & 0.025 \\
\hline Duodenal ulcer scar & $1.392(0.815-2.380)$ & 0.226 \\
\hline
\end{tabular}

Adjusted for age.

$\mathrm{Cl}$, confidence interval.

${ }^{*}$ Calculated using multiple logistic regression.

$42.4 \%$ of married Korean couples. In the analysis of the factors associated with the concurrence of $H$. pylori infection in Korean adults, the presence of an active duodenal ulcer was associated with an increased risk of conjugal infection with $H$. pylori. However, duodenal ulcer scars were not associated with concurrent $H$. pylori infection. In addition, gastric erosion was inversely associated with $H$. pylori concurrence.

The concurrence rate of $H$. pylori infection among spouses varies among the existing studies. In a UK study, $18.6 \%$ of patients in the concurrent group among 102 couples had at least one spouse with $H$. pylori infection. ${ }^{9)}$ The $H$. pylori infection concurrence rate was $33.3 \%$ in a German study, ${ }^{7)}$ which was slightly lower than that in the present study. However, in an Italian study involving 161 participants, the prevalence of $H$. pylori concurrent infection was $74.5 \%{ }^{10)}$ Additionally, the concurrence rate of $H$. pylori was $75 \%$ among 25 Indian couples, which was much higher than in the current study. ${ }^{6}$ Although the reason for these discrepancies remains unclear, it is suggested that both a heterogeneous study design and population contribute to the inconsistencies among studies worldwide.

An active duodenal ulcer was associated with an increased risk of conjugal $H$. pylori infection; however, duodenal ulcer scars were not associated with concurrent $H$. pylori infections. Because $H$. pylori infection is a well-known independent risk factor for active duodenal ulcers, ${ }^{11-14)}$ it can be inferred that participants with active duodenal ulcers had higher bacterial loads than participants free from duodenal ulcers. As a result, a partner of a patient diagnosed with a duodenal ulcer was more likely to be infected with $H$. pylori originating from his or her spouse. Moreover, our finding that duodenal ulcer scars were not associated with concurrent $H$. pylori infection indirectly supports the requirement for a distinct bacterial load to manifest spousal transmission and infection with $H$. pylori. However, we were unable to verify the exact transmission pathway of $H$. pylori or DNA types of $H$. pylori; therefore, further studies are warranted to elucidate the underlying mechanisms of this association.

Our study also showed that gastric erosion was inversely associated with concurrent $H$. pylori infection. However, previous studies evaluating the relationship between $H$. pylori infection and gastric erosion have reported inconsistent results. For instance, a study in Japan reported that $H$. pylori affects the progression of gastric precancerous lesions, glandular atrophy, and intestinal metaplasia in patients with gastric erosion. ${ }^{15)}$ In contrast, another study concluded that no significant relationship exists between $H$. pylori gastritis and gastric erosion. ${ }^{16)}$

While concurrent $H$. pylori infections were positively associated with the presence of an active duodenal ulcer, gastric erosion showed a negative association. Although it is difficult to explain the inverse association between $H$. pylori infection and duodenal ulcers and gastric erosion, several mechanisms have been hypothesized. First, the difference in intragastric acidity between erosive gastritis and $H$. pylori infection might play a role in this outcome. Erosive gastritis is linked to intragastric hyperacidity, whereas chronic $H$. pylori infection leads to an intragastric hypoacidic state by impairing the secretion of gastric acid, especially in the antrum. ${ }^{17)}$ Considering that hyperacidity causes erosion of gastric surfaces, it is plausible to infer that erosive gastritis is less likely to occur in the presence of a chronic H. pylori infection, which is associated with decreased intragastric acidity. In a previous study, H. pylori infection did not show any predisposition to erosive gastritis. ${ }^{18)}$ Another possible explanation is that people with an $H$. pylori infection are more likely to take anti-acids, such as protein pump inhibitors, to control gastrointestinal symptoms. Therefore, a low prevalence of gastric erosion may be observed among the members of the concurrent $H$. pylori infection group. Furthermore, other factors, such as psychological and physiological stress caused by erosive gastritis, may influence the manifestation of this finding.

Overall, this study has several limitations. First, since this study was conducted as a cross-sectional study, caution must be exercised when drawing conclusions regarding the causality of exposure and outcome. Moreover, as the study population was solely composed of Korean patients who underwent health check-ups, the observed findings may not be generalizable to other populations. Third, there was no evaluation of the ribosomal RNA gene patterns of $H$. pylori strains, so the spousal concurrence of $H$. pylori strains could not be verified. Finally, information on previous $H$. pylori eradication and the forms of physical contact between spouses that could possibly influence the concurrence of infection were not accounted for in the analysis owing to the lack of existing data. However, this study is the first to estimate the factors associated with concurrent $H$. pylori infections among Korean spouses.

In conclusion, spousal transmission and the concurrence of $H$. pylori infections were negatively associated with age and gastric erosion; however, they showed a positive correlation with duodenal ulcers. These results were consistent, even after correcting for age. This can be seen as a meaningful result because it elucidates spousal transmission 
of the disease, given that $H$. pylori-positive spouses act as a risk factor for spousal concurrence. Further studies are needed to determine the role of spousal transmission in intrafamilial transmission of $H$. pylori infection and the greater risk factors for spousal concurrence.

\section{CONFLICT OF INTEREST}

No potential conflict of interest relevant to this article was reported.

\section{ORCID}

Jiwoong Choi: https://orcid.org/0000-0002-5302-1628

Jihun Kang: https://orcid.org/0000-0002-2263-9054

\section{REFERENCES}

1. Kusters JG, van Vliet AH, Kuipers EJ. Pathogenesis of Helicobacter pylori infection. Clin Microbiol Rev 2006;19:449-90.

2. Hooi JK, Lai WY, Ng WK, Suen MM, Underwood FE, Tanyingoh D, et al. Global prevalence of Helicobacter pylori infection: systematic review and meta- analysis. Gastroenterology 2017;153:420-9.

3. Graham DY. History of Helicobacter pylori, duodenal ulcer, gastric ulcer and gastric cancer. World J Gastroenterol 2014;20:5191-204.

4. Magalhaes Queiroz DM, Luzza F. Epidemiology of Helicobacter pylori infection. Helicobacter 2006;11 Suppl 1:1-5.

5. Delport W, van der Merwe SW. The transmission of Helicobacter pylori: the effects of analysis method and study population on inference. Best Pract Res Clin Gastroenterol 2007;21:215-36.

6. Singh V, Trikha B, Vaiphei K, Nain CK, Thennarasu K, Singh K. Helicobacter pylori: evidence for spouse-to-spouse transmission. J Gastroenterol Hepatol 1999;14:519-22.

7. Brenner H, Rothenbacher D, Bode G, Dieudonne P, Adler G. Active infection with Helicobacter pylori in healthy couples. Epidemiol Infect 1999;122:91-5.
8. Brenner H, Weyermann M, Rothenbacher D. Clustering of Helicobacter pylori infection in couples: differences between high- and lowprevalence population groups. Ann Epidemiol 2006;16:516-20.

9. Stone MA, Taub N, Barnett DB, Mayberry JF. Increased risk of infection with Helicobacter pylori in spouses of infected subjects: observations in a general population sample from the UK. Hepatogastroenterology 2000;47:433-6.

10. Sgambato D, Visciola G, Ferrante E, Miranda A, Romano L, Tuccillo C, et al. Prevalence of Helicobacter pylori infection in sexual partners of H. pylori-infected subjects: role of gastroesophageal reflux. United European Gastroenterol J 2018;6:1470-6.

11. Hopkins RJ, Girardi LS, Turney EA. Relationship between Helicobacter pylori eradication and reduced duodenal and gastric ulcer recurrence: a review. Gastroenterology 1996;110:1244-52.

12. Parente F, Maconi G, Sangaletti O, Minguzzi M, Vago L, Rossi E, et al. Prevalence of Helicobacter pylori infection and related gastroduodenal lesions in spouses of Helicobacter pylori positive patients with duodenal ulcer. Gut 1996;39:629-33.

13. Hopkins RJ, Morris JG Jr. Helicobacter pylori: the missing link in perspective. Am J Med 1994;97:265-77.

14. Graham DY. Campylobacter pylori and peptic ulcer disease. Gastroenterology 1989;96(2 Pt 2 Suppl):615-25.

15. Zhang C, Yamada N, Wu YL, Wen M, Matsuhisa T, Matsukura N. Helicobacter pylori infection, glandular atrophy and intestinal metaplasia in superficial gastritis, gastric erosion, erosive gastritis, gastric ulcer and early gastric cancer. World J Gastroenterol 2005;11:791-6.

16. Maguilnik I, Neumann WL, Sonnenberg A, Genta RM. Reactive gastropathy is associated with inflammatory conditions throughout the gastrointestinal tract. Aliment Pharmacol Ther 2012;36:736-43.

17. Lee SY. Endoscopic gastritis, serum pepsinogen assay, and Helicobacter pylori infection. Korean J Intern Med 2016;31:835-44.

18. Kate V, Ananthakrishnan N, Badrinath S, Amarnath SK, Balamurugan M, Ratnakar C. Helicobacter pylori infection and erosive gastritis. J Assoc Physicians India 1998;46:436-7. 